\title{
Kernos
}

Revue internationale et pluridisciplinaire de religion grecque antique

$15 \mid 2002$

Varia

\section{La généalogie grecque parmi les Celtes}

\section{Maria de Henar Velasco López}

URL : http://journals.openedition.org/kernos/1388

DOI : 10.4000/kernos.1388

ISSN : 2034-7871

\section{Éditeur}

Centre international d'étude de la religion grecque antique

\section{Édition imprimée}

Date de publication : 1 janvier 2002

ISSN : 0776-3824

\section{Référence électronique}

Maria de Henar Velasco López, «La généalogie grecque parmi les Celtes », Kernos [En ligne], 15 | 2002, mis en ligne le 21 avril 2011, consulté le 03 mai 2019. URL : http://journals.openedition.org/ kernos/1388; DOI : 10.4000/kernos.1388 


\section{La généalogie grecque parmi les Celtes}

Les avancées de l'archéologie permettent aujourd'hui de relever l'existence de relations gréco-celtes dès le vir siècle av. J.-C., et même le vir ${ }^{\mathrm{e}}$. Cependant ni les services à vin, ni les amphores, pas même le beau cratère de Vix ne peuvent répondre à la question la plus fondamentale formulée par les Grecs aux princes celtes lors de leurs cérémonies d'hospitalité : qui sont vos parents? De quelle lignée êtes-vous tous issus? Bien que leurs relations fussent courantes et amicales et propices à l'échange d'objets et de récits, aucun témoignage direct des Celtes et des Grecs de cette époque ne nous est parvenu. Le monde est complètement différent au moment où César ( $D e$ bello gallico IV, 18, 1) raconte: Galli se omnes a Dite patre prognatos praedicant idque $a b$ druidibus proditum dicunt. C'est une assertion étonnamment proche de ce que disent les sources médiévales irlandaises à propos de Donn ${ }^{1}$. Mais plusieurs siècles séparent ces deux époques. Les Celtes, avec qui les Grecs nouèrent des liens amicaux, n'ont pas tous été semblables dans le temps, alors que la meilleure connaissance et les mouvements des Celtes vers le noyau méditerranéen entraînèrent un changement d'attitude gréco-latine ${ }^{2}$.

Néanmoins leurs liens amicaux ${ }^{3}$ perdurent jusqu'au Iv ${ }^{\mathrm{e}}$ siècle av. J.-C. Le plus ancien témoignage en la matière s'en fait l'écho. Aristote ${ }^{4}$ relate la

1 Vid. Ma.H. Velasco López, El paisaje del más allá. El tema del prado verde en la escatología indoeuropea, Valladolid, Universidad de Valladolid, 2001, p. 283 sq.

2 Strabon (IV, 4, 6) sait que des éléments conservés par Éphore ne sont plus d'actualité.

3 Vid. EpHor. FGrH $70 \mathrm{~F} 131 \mathrm{a}$ et b Jacoby. Pour l'étude des sources grecques sur les Celtes: D. Rankin, Celts and the Classical World, London, 1996" (London, 1987) et "The Celts through Classical Eyes", in M. Green (éd.), The Celtic World, London/New York, Routledge, 1995, p. 21-33; G. Doвesch, "Les sources littéraires", in Les Celtes, Milano, 1991, p. 35-41; Ph. M. Freeman, "The earliest Greek sources on the Celts", EC 32 (1996), p. 11-48. La meilleure compilation de sources est l'œuvre de I. ZWICKer, Fontes Historiae Religionis Celticae, in C. CLEMEN (éd.), Fontes Historiae religionum ex auctoribus graecis et latinis collectos, Fasciculus V, Berolini, 1934, I, Bonnae, 1935, II, Bonnae, 1936, III.

4 Athénée, XIII, 576 a-b. Pour des détails sur la transmission du texte, vid. D.'Pralon, "La légende de la fondation de Marseille", in M. Bats, G. Bertucchi, G. Conges, H. TreZINY (éds), Marseille grecque et la Gaule. Actes du Colloque international d'Histoire et d'Arcbéologie et du $V^{e}$ Congrès archéologique de Gaule méridionale (Marseille, 18-23 
fondation de Massalia (Marseille) en ces termes : Euxénos, le Phocéen, était l'hôte du roi et était présent, par hasard, le jour même de la noce de la fille de celui-ci. Après le banquet, celle-ci devait entrer et remettre une patère pleine de vin au prétendant de son choix. Elle choisit Euxénos et de leur union allait naître Protis, dont descend toute la lignée des Protiades.

On peut heureusement mettre en contraste ce récit avec celui rapporté par Justin ${ }^{5}$ (III ${ }^{\mathrm{e}}$ s. ap. J.-C.) sept siècles plus tard. Les variations sont minimes : il y a deux chefs Phocéens, on précise qu'il s'agit du roi des Ségobrigues, le nom de la fille change et c'est de l'eau qu'elle offre. La plus intéressante est que Protis n'est pas ici le fils mais le mari phocéen. Ne s'agissant pas d'un calque du texte d'Aristote, on doit par conséquent rejeter l'hypothèse d'une dépendance intertextuelle et penser plutôt à l'existence d'une légende sur la fondation de Marseille, connue indépendamment. Quant au crédit qu'on lui accorde, c'est une autre question.

Massalia est fondée aux environs de 600 av. J.-C. Aristote écrit au $\mathrm{Iv}^{\mathrm{e}}$ siècle av. J.-C. Il est probable qu'il n'ait pas parlé avec ses habitants, mais on peut supposer qu'il a vérifié les meilleures sources à sa disposition. Cette citation est tirée de sa Constitution des Massaliotes, mais on ne doit pas oublier qu'il a écrit un autre ouvrage à propos des mœurs barbares ${ }^{6}$. Il ne faut pas non plus écarter la possibilité d'un contact direct si l'on pense aux séjours de Platon dans une Sicile si fréquentée par des mercenaires celtes également présents à la cour macédonienne à laquelle Aristote lui-même est lié. La description de la noce, si différente des coutumes grecques, semble évidemment authentique, d'autant plus qu'elle coïncide avec celle de Justin. Celuici, c'est important de le noter, fait le résumé de l'œuvre de Trogue-Pompée, une excellente source, parce que tout comme Voconce, originaire de la région, il devait être très bien informé (il nous donne le nom celte du peuple, segobrigi). C'est précisément les changements de noms dans sa narration qui dissipent les soupçons de conte populaire, éveillés par le récit d'Aristote : le nom évocateur d'Euxénos disparaît, et d'autre part une référence indirecte de Plutarque (Solon, 2, 6-7) suggère que Protis fut un colonisateur, faisant du commerce avec les Celtes autour du Rhône ${ }^{7}$.

novembre 1990), Aix-en-Provence, A.D.A.M. éditions, 1992 (Collection Études Massaliètes, 3), p. 51-56.

5 XIIII 3, 8 sq. À propos de Trogue-Pompée, vid. Rankin, o.c. (n. 3) p. 40, ainsi que la préface de la traduction par J. CAstro SánChez, Madrid, Gredos, 1995 et J.M. AlonsoNúÑez, "Trogue-Pompée et Massilia (Justin, Epitoma XLIII, 3, 4-XLIII, 5, 10)", Latomus 53 (1994), p. 110-117.

6 Vid. Dobesch, l.c. (n. 3) p. 35 sq.; Freeman l.c. (n. 3) p. 24 n. 49 et p. 42 sq.; Rankin, o.c. (n. 3) p. 46 et p. 54.

7 JACOBY (n. 377 au commentaire de Timée F 70-72) souligne cette différence entre la tradition romancée d'Aristote et l'historique de Trogue-Pompée. D'autre part, D. et Y. Roman (Histoire de la Gaule. vie siècle av. J.-C. - $I^{\text {r }}$ siècle ap. J.-C., Paris, Fayard, 1997, p. 264 n. 139) remarquent que Protis est peut-être simplement $\pi \rho \hat{\tau} \tau$ os. Cette lecture appa- 
Les conclusions tirées de ces textes ont été très diverses : la première correspond à l'interprétation du mythe du point de vue de la colonisation ${ }^{8}$, la seconde à l'attribution de parallèles avec d'autres histoires d'amour dans lesquelles c'est la femme qui choisit'; une troisième y voit un premier témoignage du mythe de la souveraineté, très bien étudié dans les sources irlandaises, l'iconographie et l'épigraphie gauloises, selon lequel la liaison du roi et de sa terre s'exprime à travers le mariage du souverain avec la déesse éponyme du territoire ${ }^{10}$.

On ne peut pas étudier ici en détails ces propositions. Mais il convient de situer le récit dans son contexte : l'existence de rẹlations courantes entre les Grecs et les Celtes, qui habitent leurs oppida et conservent leurs propres

raît dans un des manuscrits de l'œuvre de Plutarque citée auparavant, mais il s'agit d'une lecture secunda manu, à mon avis faite par un scribe qui corrige le texte.

8 Ainsi pour M. Bats et P. MOREL, cités par Alonso-NúÑEZ, l.c. (n. 5), p. 111 n. 5 et p. $112 \mathrm{n} .6$, le mariage symbolise la fusion entre indigènes et Grecs, qui, pour s'installer, avaient besoin de prendre en mariage lés filles du pays. Cela se rattache au débat sur la participation des femmes à la colonisation. Il faut souligner le texte de Strabo (IV, 1, 4) qui, dans son récit plus proche à la version "historique " d'une colonisation, mentionne une femme de haute lignée parmi les phocéens qui arrivent à Massalia.

9 Cette correspondance est déjà établie par ATHÉNÉE (XIII, 36, 576 a-b), qui avant le récit d'Aristote en raconte un autre, provenant de Charès de Mytilène, historien d'Alexandre le Grand. Cependant, les différences sont plus nombreuses que les similitudes. Il est très probable que, comme Pralon, l.c. (n. 4), et Roman, o.c. (n. 7), p. 264 sq., le soulignent, il y ait dans ces récits l'écho de traditions indo-européennes plus anciennes. Mais l'approfondissement de ce thème impliquerait une autre ligne de recherche. Il faut noter ici que, contrairement au récit d'Aristote, l'amour pour une personne apparue en songe est le motif principal du récit de Charès ainsi que celui de Nala et Damayanti parmi les Indiens, cela correspond mieux à l'histoire irlandaise d'Óengus (Fr. SHAw, The Dream of Oengus (Aislinge Oenguso). An Old Irish text critically restored and edited with notes and glossary, Dublin, 1934, cf. le c.r. par J. VeNDrYes, EC 1 [1936], 159-162). Les parallèles cités par Pralon concernent seulement le choix de la femme., Ils aident à identifier le fond ultime de ces traditions. Mais il est important ici de souligner la fonction assignée par les Celtes à un tel choix, dans la mesure où les textes antiques et médiévaux le reflètent.

10 A mon avis, le fait que dans le cas des couples en sculpture le personnage masculin reçoive parfois un nom romain, mais que la figure féminine soit toujours une déesse indigène est très significatif (vid. M. Herber, "Godddes and King: The Sacred Marriage in Early Ireland", in L.O. Fradenburg (éd.), Women and sovereignity, Edinburgh, 1992, p. 264-275, p. 265). Sur ce thème on citera : P. Mac CanA, "Aspects of the theme of king and goddess in Irish literature", EC 7 (1955-56), p. 76-114 et p. 356-413; 8 (1958-59/59-65) et Celtic Mythology, New York, 1985, p. 114-121; K. MCCone, Pagan Past and Christian Present in Early Irish Literature, Maynooth, 1991, p. 109 sq.; pour le développement irlandais du sujet, vid. R.A. BrEanAch, "The Lady and the King, a theme of Irish Literature", Studies 12 (1953), p. 321-36; plus récemment, J.P. MACKEY, "Mythical Past and Political Present: A case-study of the Irish Myth of the Sovereignity", $Z C P 51$ (1999), p. 66-84 et M. Aldhousse-Green, "Pagan Celtic iconography and the concept of sacral kingship", ZCP 52 (2001), p. 102-117; cf. FreEman, l.c. (n. 3), p. 15 n. 12. 
mœurs aux portes mêmes de Marseille et jusqu'à une époque très tardive ${ }^{11}$. Ce contexte rend très vraisemblable l'hypothèse de l'adaptation d'un mythe celtique, grâce auquel les Celtes sont désormais apparentés aux Grecs à travers une généalogie réelle, humaine, qu'Aristote limite à la lignée des Protiades.

Ceci est important car les autres sources qui traitent des origines des Celtes ne connaissent pas cette restriction. Pour elles, il s'agit d'un ancêtre commun à tous les Celtes avec un lien de parenté mythologique. Les principaux ascendants sont au nombre de deux : Héraclès et Galatée.

On centrera d'abord l'attention sur Galatée. Une version peu connue de ses amours avec Polyphème affirme qu'ils eurent un fils, Galatos, selon Timée $^{12}$ (III ${ }^{\mathrm{e}}-\mathrm{II}^{\mathrm{e}}$ s. av. J.-C.), ou, selon Appien ${ }^{13}\left(\mathrm{I}^{\mathrm{er}}-\mathrm{II}^{\mathrm{e}}\right.$ s. ap. J.-C.), trois fils, Celtos, Illyrios et Galas, convertis en héros éponymes de leurs peuples. Les sources qui relatent cette version sont plus tardives que celles étudiées auparavant, bien qu'il faille considérer la possibilité de remonter à des

11 Vid. O. Büchenschürz, "The Celts in France", in Green, o.c. (n. 3), p. 552-580, p. 573 sq. et plus spécifiquement, J.-P. Morel, "Les Grecs et la Gaule", in Les Grecs et l'Occident. Actes du Colloque de la Villa "Kérylos" (1991), Rome, 1995, p. 41-69. On constate une dépendance réciproque entre les influences grecques et indigènes (Celtes et Ligures), qui dépasse le territoire limitrophe et pénètre vers l'intérieur (les routes de la culture hallstattique). En outre, les Celtes ne furent pas tous identiques et ils évoluèrent au fil du temps. Cette question a été bien soulignée par G. López Monteagudo ("La religión céltica, gala y galo-romana", in Historia de las religiones de la Europa Antigua, Madrid, 1994, p. "421-488). Dans son article on peut trouver diverses preuves de la survivance de cultes indigènes aux environs de la métropole grecque, ainsi, un bois sacré celtique près de Marseille (Lucain, Pharsale III, 399 sq.), habité par les dieux barbares; César le fait abattre au $I^{\text {er }}$ s. av. J.-C.

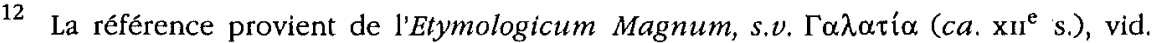
JACOBy Timée F 69. Il convient ici de se rappeler de l'origine sicilienne de l'auteur, qu'il a étudié à Athènes avec un disciple d'Isocrate, autour duquel s'était formé un intéressant cercle de géographes voyageurs (Fremman, l.c. [n. 3], p. 34 sq.), et qu'il a fréquenté des membres de l'école péripatéticienne, si encline à la rationalisation des mythes et à l'élaboration des étymologies, bien que sérieuse dans la collecte des renseignements.

13 Historia romana, Ilírica 2, Le fond du texte démontre la consultation de divers mythographes parmi lesquels il choisit la version qu'il préfère, bien qu'il renvoie aux auteurs qui s'occupent de périodes plus reculées, une formule utilisée quand il aborde des sujets secondaires qu'il ne va pas approfondir, ce qui est peut-être une manière de cacher son ignorance. Bien qu'il soit difficile de préciser ses sources (vid. avec bibliographie la traduction par A. SANCHo Royo, Madrid, Gredos, 1980), dans ce livre il cite les mémoires d'Auguste et il ne faut pas négliger la dépendance de Timagène d'Alexandrie, historien de l'époque augustenne, très cité concernant les Celtes. En outre Polybe est mentionné par Appien et il a probablement utilisé l'œuuvre de Posidonios. Évidemment il n'invente pas cette généalogie, très éloignée de la plus traditionnelle et postérieure à l'élaboration de l'étymologie Galates-Galatée ( $c f$. WeIcker, s.v. Illyrios, $R E$ IX 1 [1914], col. 1088-1089). Pour la différence entre Celtes et Galates, infra n. 17. 
périodes plus anciennes ${ }^{14}$. Il est indispensable de considérer les limites chronologiques de la légende de Polyphème et Galatée.

Le $\mathrm{v}^{\mathrm{e}}$ siècle av. J.-C. doit être considéré comme la date post quem pour l'établissement des versions de leurs amours, pas toujours contrariées, bien que ce soient des textes tardifs qui évoquent l'amour partagé, confirmé dans des représentations iconographiques et qui, en ce qui nous concerne, donnera lieu à une descendance ${ }^{15}$. L'élaboration mythographique semble en tous cas évidente. L'unique raison de relier cette légende, dans l'une de ses versions les moins connues, au destin des Celtes est la correspondance entre les noms de Galatée et de Galatos. Le fait qu'il s'agisse d'une légende sicilienne désigne cette île comme la localisation d'origine faite par son premier auteur: une Sicile où l'on trouve très souvent des mercenaires celtes dès l'époque de Denys $I^{\text {er }}$ de Syracuse (430-367 av. J.-C. $)^{16}$. Le fait même du jeu étymologique a son importance (quoique Appien distingue Celtos et Galas, le point de départ est le même) : la dénomination Galatai, différente de Keltoi, Keltine, n'apparaît pas avant Timée ${ }^{17}$, au III ${ }^{\mathrm{e}}$ siècle av. J.-C. Il semble donc difficile de remonter au-delà de cette date.

14 On ne voit pas comment un mythographe de la Renaissance pourrait connaître un prétendu témoignage de Bacchylide (Natalis Comes, Myth. IX, 8, p. 975 Genev., cité par JAcoBy dans son commentaire à Timée $\mathrm{F}$ 69): Dicitur Polypbemus non modo amasse Galateam, sed etiam Galatum ex ea suscepisse, ut testatus est Bacchylides, cum quidam Cellum etiam Polypbemi filium fuisse inquiant, a quo dicti sunt Celtae, et Illyrium, a quo Illyris, et Henetum, ut quidam uoluerunt, a quo regio postea Venetia, et Papblagonum, ut ait Dercyllus in libro De nominibus urbium et locorum). Pour R. PFEIfFer dans son édition de Callimaque (Oxford, 1949, reimpr. 1985, vol. I, p. 304 sq.), Natale Conti a créé cette tromperie à partir de l'Etymologicum-Magnum. Callimaque, d'autre part, offre seulement un argument ex silentio : le fragment 379 est attribué à sa Galatée pour le nom du chef gaulois Brennus. Un Callimaque prolifique, directeur de la Bibliothèque d'Alexandrie, auteur érudit de plusieurs œuvres dédiées aux peuples étrangers, leurs dénominations ethniques, les fondations, ainsi que collectionneur de merveilles présentait le profil approprié. Mais, à défaut de nouveaux témoignages (dans l'Hymne 4, $173 \mathrm{sq}$. et 184, il considère les Celtes comme descendants des Titans, $c f$. des représentations des Celtes dans la sculpture hellénistique), la prudence est de rigueur (cf. RaNkin, l.c. [n. 3], p. 22).

15 Deux auteurs de l'école péripatéticienne, entre le $\mathrm{IV}^{\mathrm{e}}$ et le $11 \mathrm{I}^{\mathrm{e}} \mathrm{s}$. av. J.-C., nous informent sur le contenu du poème de Philoxène de Cythère, qui au $v^{e} s$. av. J.-C. aurait introduit un changement radical dans la personnalité de Polyphème. En ce qui nous concerne, le fait qu'il se soit appuyé sur des traditions autochtones de la Sicile ou qu'il ait forgé cela ex nibilo en adaptant sa propre biographie ne nous intéresse pas. Pour les détails, vid. les articles Polypbemos et Galateia in LIMC Supplementum I et vol. V, 1 p. 1000-1005. On accordera une attention particulière aux représentations dans lesquelles Polyphème reçoit une lettre de Galatée et celles de leur embrassement amoureux (V, 2, p. 630).

16 Xén., Hell. VII, 1, 20 et 31. Vid. Freeman, l.c. (n. 3), p. 20; Rankin, o.c. (n. 3), p. 45 et DoBesch, l.c. (n. 3), p. 36 sq.).

17 Pour cette question, vid, Roman, o.c. (n. 7), p. 209 sq. 
Sur le même jeu étymologique est fondé le récit de Parthénius de Nicée (ca. 70 av. J.-C. Narrationes amatoriae XXX), mais avec une différence substantielle : la protagoniste est la jeune Celtine. Amoureuse d'un Héraclès revenant d'Érythie avec les bœufs de Géryon et déambulant dans les terres celtiques, Celtine cache ses bœufs pour l'obliger à s'unir à elle. De leur union naîtra Celtos qui donnera son nom à tous les Celtes ${ }^{18}$. Au $\mathrm{I}^{\text {er }}$ siècle av. J.-C. également, Diodore fait entrer Héraclès dans la Celtique et lui fait fonder la cité d'Alésia ${ }^{19}$ (IV, 19) : une belle et robuste demoiselle, fille du souverain local, qui jusqu'alors avait rejeté tous ses prétendants, et que la puissance et la supériorité d'Héraclès émerveillent. De leur union naîtra un fils, Galatos.

Il faut souligner quelques aspects : les personnages celtes, soit ne reçoivent pas de noms, soit ceux-ci sont des éponymes (Bretanos, Celtine); la jeune fille joue un rôle important, semblable à celui de l'héroïne dans le récit de Marseille, c'est elle qui choisit non pas un hôte quelconque (Euxénos, Protis) mais bien Héraclès ${ }^{20}$. Le héros barbare et civilisateur, fils de Zeus, inlassable voyageur et amant infatigable ${ }^{21}$, père de nombreuses progénitures grâce auxquelles il établit des rapports avec de multiples territoires ${ }^{22}$ qui renforcent son panhellénisme et son énorme popularité, était le candidat parfait pour faire entrer les Celtes dans la mythologie grecque. Pour les colonisateurs, le mariage d'Héraclès avec la fille du roi confère une sanction

18 Des sources plus tardives (ZwICKER, o.c. [n. 3], III p. 258 et p. 271) mentionnent Celto, fille de Bretanos. La capture des bœufs de Géryon ne figure plus, mais il y a une épreuve avec l'arc ( $c f$. HÉr., IV, 8 et n. 25).

19 Pour d'autres traces de mythologie celtique dissimulés dans des récits de faits supposés réels, vid. López Monteagudo, l.c. (n. 11), p. 449, avec des références à d'autres légendes de fondation de cités. Pour le fond historique, vid. Roman, o.c. (n. 7), p. 196 et C. JourdaIn-ANNEQuin, "Héraclès en Occident, mythe et histoire", DHA 8 (1982), p. 227-282.

20 On soulignera ici seulement quelques travaux généraux : les articles toujours utiles de la $R E$ (VIII [1912], col. 516 sq.; VIII [1912], col. 550 sq.; Suppl. III [1918], col. 910 sq.; Suppl. 14 [1974], col. 137-196) et du Neue Pauly. Pour les représentations : A. FurTẄ̈NGLER, in W.H. Roscher, Ausfübrlicbes Lexikon..., Leipzig, 1909-1915, s.v. Herakles, I, 2, col. 2135-2252; F. Bommer, Heracles. The Twelve Labors of the Hero in Ancient Art and Literature, New York, 1986 (Köln, 1979); R. VollKommer, Herakles in the Art of Classical Greece, Oxford, 1988; J. Bonrman, O. Palagia, S. Woodford, "Herakles", LIMC IV, 1 (1988), p. 728-838 et V, 1 (1990), p. 1-196 ainsi que L.J. BALmasedA, "Hercules (in peripheria occidentali)", LIMC V, 1 (1990), p. 253-262.

21 RE Suppl. III (1918), col. 1090-1095; LIMC IV, 1 (1988), p. 821-824. Grâce à ses relations avec des femmes, il devient le fondateur de diverses généalogies royales réparties à travers tout le monde antique et son nom est utilisé pour légitimer le pouvoir politique, également dans les colonies (Vollkommer, o.c. [n. 20], p. 88 sq.)

22 Parmi les cités qui portent son nom (vid. RE VIII [1912], s.v. Herakleia et Vollkommer, o.c. [n. 20], p. 101 et p. 107), il faut remarquer une cité dans les bouches du Rhône, dans un lieu où des inscriptions grecques ont été trouvées ainsi que des traces du culte à l'Héraclès phénicien (RE VIII [1912], col. 609 et s.v. Heraclea 2). 
divine au fait de s'emparer d'une terre étrangère ${ }^{23}$. Il s'agit pour eux d'une occupation pacifique, car les ancêtres de ce peuple sont aussi grecs. Pour les autochtones, ce rattachement généalogique constitue également un honneur, établissant une alliance avec les Grecs tant admirés ${ }^{24}$. On peut de ce point de vue envisager cette version comme une réinterprétation du récit de la fondation de Marseille ${ }^{25}$ : on retrouve l'identification de la jeune fille avec le territoire, particularité essentielle du mythe celtique de souveraineté; le prétendant n'est plus un colonisateur réel ou romancé, mais le colonisateur par autonomase, Héraclès; la généalogie ne se limite pas à la lignée d'une colonie grecque ${ }^{26}$, bien au contraire, elle englobe toute la Celtique; le fait que Diodore situe l'action à Alésia a une explication concrète, très bien étudiée par C. Jourdain-Annequin ${ }^{27}$ : justifier ainsi la conquête de César, nouvel Héraclès de la Gaule.

Or, ni Diodore ni Parthénius ne sont des créateurs ex nibilo. Ils ne sont pas non plus les seuls qui, au $\mathrm{I}^{\mathrm{er}}$ siècle av. J.-C., attribuent la paternité des Celtes à Héraclès. Cependant, les autres récits présentent des variations : pour Denys d'Halicarnasse (XIV, 1, 3) Héraclès et Astéropè, la fille d'Atlas, ont eu deux fils, Ibèros et Celtos ${ }^{28}$; Ammien, dans un passage où il cite Timagène,

23 Ce fait pouvait être considéré comme un crime, A.J. Graham, "The western Greeks", in J. Boardman, N.G.L. Hammond (éds.), Cambridge Ancient History, Cambridge, $1992\left(1982^{2}\right)$ III, 3, p. 145.

24 Il faut remarquer l'importance de la généalogie parmi les Celtes : comme dans Homère, les défis entre des champions ont des références spécifiques (RANKIN, o.c. [n. 3], p. 68); dans l'Irlande médiévale, l'élaboration de généalogies est une occupation prioritaire (E. O'CurRy, Lectures on the Manuscript Materials of Ancient Irish History, Dublin, 1995 [1861], p. 203 sq.) et l'ascendance grecque est toujours cause de vantardise (W.B. Stanford, Ireland and the Classical Tradition, Dublin, 1976, p. 79).

25

Gf. l'allusion dans ce sens de C. Jourdain-ANnequin, Héraclès aux portes du soir, Paris, 1989 , p. 311 , bien que je ne partage pas son évaluation du texte d'Hérodote (IV, 8). La comparaison invite à voir un fond commun, mais les différences sont trop importantes pour considérer une réélaboration à partir du texte hérodotéen (ainsi L. PRELLER, Griecbiscbe Mytbologie, Zweiter Band. Die Heroen (Die Griecbiscbe Heldensage), ern. von C. Robert, Hildesheim, 1995 (Berlin, 1921), p. 476 et p. 482, tandis que GruPPE (in $R E$ Suppl. III [1918], col. 998) a soutenu l'opinion contraire.

26 Le parti hellénisé fut favorisé lors de la destruction de l'oppidum d'Entremont par les Romains (Jourdain-ANNEquin, o.c. [n. 25], p. 638 n. 817). On entrevoit ainsi une des causes de l'intérêt des indigènes pour une ascendance grecque, qui s'offre ici à tous les Celtes sans restriction.

27 o.c. (n. 25), p. 245 sq., p. 309 sq., p. 638 sq. Héraclès, remarquons-le ( $c f$. p. 313), instaure un royaume de justice, un aspect aussi souligné dans le récit d'Ammien Marcellin (XV, 9, $2 s q$.). Il y a ici une différence très importante par rapport aux autres textes (Parthénius, Denys d'Halicarnasse ainsi que le récit de la fondation de Marseille ou la généalogie à partir de Galatée), il n'y a plus un contexte de rapports amicaux, mais l'intérêt impérialiste de Rome, appuyé par Diodore.

28 Cf. Eust., In Dionysium Periegeten, 281 (Zwicker, o.c. [n. 3], vol. III, p. 273), bien que $i b .69$ l'ascendance des Galates soit attribuée à Apollon. Pour Astéropè, vid. Etymo-

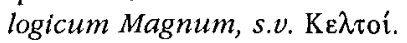


recueille plusieurs versions mais c'est à la suivante, racontée par les habitants de ces contrées et que lui-même a vue gravée sur leurs monuments, que l'on accordera le plus de crédit: Héraclès, après avoir vaincu les cruels tyrans Géryon et Tauriscus qui dévastaient l'Espagne et les Gaules, eut, de ses relations avec des femmes nobles, plusieurs enfants qui appelèrent de leur propre nom les régions qu'ils gouvernaient. Ces monuments ont malheureusement disparu, mais on peut lire sur quelques inscriptions latines le nom d'Héraclès suivi d'une épithète celtique qui correspond soit au nom d'un peuple, soit au nom d'un territoire ${ }^{29}$. Cette correspondance est caractéristique de la théonymie celtique, selon ce que l'on en sait. $A$ mon avis, ces inscriptions peuvent être considérés comme la confirmation archéologique d'une généalogie héracléenne des peuples celtiques ${ }^{30}$.

Avec quelle précision peut-on déterminer l'époque où commença cette relation ? La première date post quem correspond naturellement à la localisation de l'aventure de Géryon en Extrême-Occident (selon Hésiode au-delà du fleuve Océan, selon Stésichore face à l'embouchure du fleuve Tartessos, le Guadalquivir) $^{31}$. Les informations que nous fournissent les auteurs grecs s'occupant de ce thème entre le $\mathrm{vII}^{\mathrm{e}}$ et le $\mathrm{v}^{\mathrm{e}}$ siècle av. J.-C. (Pisandre de Camiros, Stésichore, Panyassis) ne nous permettent pas de déterminer si, lors de son retour, Héraclès traversa ou non l'Europe barbare. Tout va dans le sens d'un développement progressif des premières rencontres du voyage. Eschyle $^{32}$ est le premier à mentioner l'affrontement avec les Ligures. En Ligurie, selon l'auteur de la Bibliothèque (II, 106 sq.), Héraclès tua deux fils de Poséidon qui tentait de lui dérober ses bœufs. C'est la même action, rappelons-le, que Parthénius attribue à Celtine.

Il est donc très probable que l'épisode celtique n'apparaît pas chez les mythographes du v $v^{\mathrm{e}}$ siècle av. J.-C., dont la Bibliothèque se voulait l'héri-

29 Vid. Haug, in RE VIII (1912), col. 609-612 el Peter, in Roscher's Lexikon I, 2 p. 3020 $s q$.

30 Dans le rite d'intronisation, le roi est marié symboliquement à la déesse du territoire (López Monteagudo, l.c. [n. 11], p. 440 et supra n. 10). Cela transparaît dans le récit de l'arrivée des Grecs à Marseille et à celui de l'union d'Héraclès avec la jeune fille de filiation celtique.

31 Les sources sont regroupées par Gruppe in "Die Rinder des Geryone(u)s", $R E$ Suppl. III (1918), col. 1061-1067. Cf. Preller, o.c. (n. 25) "Geryoneus" p. 465-483. J.H. CROON (The Herdsman of the Dead. Studies on some Cults, Myths and Legends of the Ancient Greek Colonization-Area, Utrecht, 1952) a bien étudié le déplacement vers l'ouest de la localisation du mythe de Géryon. Pour Jourdain-Annequin (o.c. [n. 25], p. 223 sq.), qui fait une analyse très minutieuse des sources de Diodore et Apollodore, ainsi que du fond idéologique dans leur traitement d'Héraclès, ce sont les colonisateurs d'Occident qui précisent le mythe du point de vue géographique et contribuent à son enrichissement. Cependant, elle souligne à juste titre la complexité des rapports mythehistoire (ib. p. 314 sq.).

32 Strabon, IV, $1,17=$ fr. 32 b Mette $=199$ Nauck, Vid. Preller, o.c. (n. 25), p. 473 n. 5 et supra n. 22. 
tière ${ }^{33}$. Pas de rencontre celtique donc, mais seulement leur précédent ligurien, vraisemblablement parce que les sources d'Apollodore mentionnent une côte occupée par les Ligures, et non pas par les Celtes ${ }^{34}$. La première mention d'une route héracléenne, qui conduit de l'Italie au pays des Celtes, Celtoligures et Ibères, provient d'un traité pseudoaristotélicien ${ }^{35}$. Étant donné que Diodore insiste aussi sur les avantages de cette route ${ }^{36}$, il serait raisonnable de penser qu'il s'inspire d'une source de cette époque, le $\mathrm{nI}^{\mathrm{e}}$ ou peut-être déjà le Iv ${ }^{\mathrm{e}}$ siècle av. J.-C.

Le fait que pour toute cette tradition l'éponyme soit Celtos, Celtes, Celtine, joue en faveur du rv siècle av. J.-C. Diodore, cependant, nomme « La Celtique » le territoire où Héraclès arrive et fonde Alésia, mais ensuite dénomme Galatos le fils et Galatie le pays. Cette confusion est le fruit du bilinguisme antique : on a dit auparavant que, dès le II $^{\mathrm{e}}$ siècle av. J.-C., la langue grecque utilise Galatai comme terme différent de Keltoi, mais le latin ne disposant que d'un vocable, les mots grecs Galatai et Galatia servent donc à traduire les termes latins de Galli, Gallia. Par conséquent, soit Diodore utilise la terminologie commune, appropriée à l'intention des auditeurs romains de son récit, soit cette terminologie laisse entrevoir une source postérieure au III $^{\mathrm{e}}$ siècle av. J.-C., peut-être latine ${ }^{37}$. D'autre part, la paternité de Celtine peut aussi fournir une piste chronologique : Bretanos implique

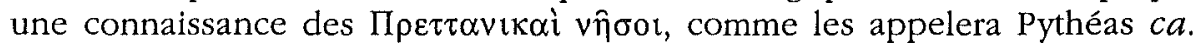
300 av. J.-C., son premier visiteur supposé e $^{38}$.

L'analyse des textes ne permet pas d'approfondir beaucoup plus. La date si tardive de leur élaboration peut attirer l'attention. Mais les connaissances sur les Celtes s'améliorent justement au Iv siècle av. J.-C. En plus, et nous l'avons vu, l'amitié envers les barbares, ainsi que le philhéllenisme (l'orgueil

33 Jourdain- ANNEQuin, o.c. (n. 25), p. 236.

34 À ce propos, Rankin, o.c. (n. 3), p. 38; Dobesch, l.c. (n. 3), p. 35; J. Déchelette, Manuel d'archéologie prébistorique, celtique et gallo-romaine, Paris, 1914, vol. IV, p. 998, p. 1004 sq.; Roman, o.c. (n. 7), p. 229 sq. et p. 207 sq.

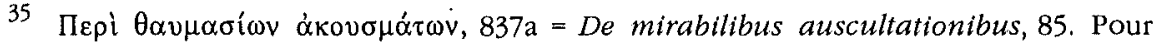
l'auteur, vid. RE Suppl. XI, col. 315 et Suppl. VII, col. $1406 \mathrm{sq}$. Avec des références aux éditions et traductions, celle de L.D. DowDALL, Oxford, 1909, et W.D. Ross, The Works of Aristotle, vol. VI, Oxford, 1913.

36 Trte Live $(V, 34,6)$ fait de l'arrivée des Phocéens et du passage des Alpes par les Celtes deux faits contemporains (vid. Roman, o.c. [n. 7] p. 207). Originaire de Padoue, il doit connaître la légende d'Héraclès. Il y fait une brève allusion : nisi de Hercule fabulis credere libet. Vid. L. PAuli, "Les Alpes à l'époque des premières migrations celtiques", in Les Celtes, p. 215-218.

37 JACOBY (II C, p. 22525 sq. et p. 21244 sq.; cf. III B, p. 5702 sq. commentaire à TiméE F 69) rejette la dépendance de Timée ou Posidonios. Le texte de Diodore V, 24 procéderait d'un manuel mythologique, identifié par quelques-uns à celui de Dionysios Scytobrachion, grammairien d'Alexandrie ( $\mathrm{I}^{\mathrm{e}}$ ou $\mathrm{I}^{\mathrm{e}}$ av. J.-C.), dont l'œuvre était inspirée par l'école d'Évhémère.

38 A. Holder, Alt-celtiscber Spracbschatz, Graz, 1961 (Leipzig, 1861), s,v. Brittani. 
barbare de se savoir descendants des Grecs) paraissent avoir dominé le contexte dans lequel ce récit fut constitué. C'est seulement à partir du $\mathrm{IV}^{\mathrm{e}}$ siècle av. J.-C. que les Celtes constituent un péril constant pour le monde grécolatin $^{39}$. Et c'est au $\mathrm{r}^{\mathrm{er}}$ siècle av. J.-C., lorsque, à la suite des campagnes de César, la Gaule devient une partie du monde romain, que les historiens profitent des récits mythologiques pour justifier une telle intervention.

La comparaison avec le développement d'autres épisodes similaires du mythe d'Héraclès pourrait nous aider à mieux comprendre ce processus. Téléphe est un roi de Mysie de la phase la plus ancienne (Homère, Cycle, Pindare); il devient un héraclide et on le rattache à la légende arcadienne au $\mathrm{v}^{\mathrm{e}}$ siècle av. J.-C. (un mythographe l'intègre à la généalogie grecque, et Sophocle le rattache à la légende arcadienne d'Augé); à la fin du III $^{\mathrm{e}}$ siècle, on note le désir de la part des Attalides de Pergame de se relier à Héraclès, rapport qui ne s'établira pas avant le $\mathrm{II}^{\mathrm{e}}$ siècle av. $\mathrm{J},-\mathrm{C}^{40}$.

Dans le cas celtique, il paraît évident que la généalogie d'Héraclès fut plus populaire que celle de Galatée. Celle-ci avait peut-être à son désavantage le fait de se baser sur une version moins étendue, selon nos connaissances, de l'amour de Polyphème et Galatée, tandis que celle d'Héraclès s'inscrivait dans l'un des cycles mythiques les plus connus et représentés, et cadrait bien avec les nombreuses aventures amoureuses et la revendication grecque traditionnelle de l'ascendance, ajoutées à l'ancrage au processus de colonisation.

Quant à l'acceptation de cette généalogie parmi les Celtes, - Ammien Marcelin nous dit que c'est la version que racontent les indigènes -, en réalité César ne mentionne pas Héraclès parmi les dieux de la Gaule. Cependant, il y a beaucoup de représentations en Gaule qui prouvent l'incorporation d'Héraclès au panthéon celtique; on a ici uniquement souligné les inscriptions. Il faut rappeler également l'Héraclès-Ogmios de Lucien (Heracles, 1 sq.), très vraisemblablement un dieu souterrain, et évaluer à sa juste valeur le lien avec l'aventure de Géryon et son caractère occidental et chthonien ${ }^{41}$. Si les Celtes assuraient qu'ils descendaient tous d'un dieu infernal, Dis Pater selon César, le contexte de la geste héracléenne pourrait présenter Héraclès,

39 Vid. Dobesch, l.c. (n. 3), p. 37; Rankin, l.c. (n. 3), p. 21; V. Kruta, "Les Celtes de la première expansion historique", in Les Celtes, p. 195-212.

40 Vid. T.S. SCHEER, Mytische Vorväter zur Bedutung griechischer Heroenmythen im selbstverständnis Kleinasiatischer Städte, München, 1993, p. 71-152; Roscher's Lexikon, V s.v. Telephos; L.R. FARnel. Greek Hero Cults and Ideas of Immortality, Chicago, 1995 (Oxford, 1921), p. 135 sq., à ce sujet on pourra consulter également d'autres exemples d'Héraclès pénétrant dans les traditions de peuples non helléniques, se combinant avec les mythes et les cultes locaux.

41 Pour Ogmios, vid. par exemple B. MA1ER, Lexikon der keltiscben Religion und Kultur, Stuttgart, 1994, s.v. Pour les aspects chthoniens d'Héraclès, LIMC V, 1 s.v. Hercules (in peripheria occidentali), p. $261 \mathrm{sq}$. Son rapport aux eaux thermales est aussi significatif (López Monteagudo, l.c. (n. 11) p. 431, 433, 442, 456; Vollkommer, o.c. (n. 20) p. 97). Pour Géryon, vid. Preller, o.c. (n. 25), p. 465; Gruppe, in RE Suppl. III (1918), col. 1064; E. VIsser, s.v. Geryoneus, Neue Pauly, IV (1998), p. 981-982. 
aux yeux des Celtes, comme un bon candidat pour figurer comme un de leurs ancêtres mythiques. De ce point de vue, la généalogie héracléenne ajusta peut-être les traditions autochtones (mythe de la souveraineté, anthropogonie) au monde grec. Ceci fut possible grâce à l'extraordinaire habilité grecque pour transformer, développer et rationaliser le mythe. Celui-ci a servi à justifier la colonisation et l'intégration des Celtes à la civilisation classique.

Ainsi s'ouvrait une voie que suivront les futures nations du Moyen-Âge afin de se dépouiller de leur barbarie. Hercule, sur le chemin de l'Espagne, s'arrêtera dans le pays de la Bourgogne pour épouser une belle et noble dame, Alise (union dont seront issus les princes de Bourgogne). En l'an 600 on se souviendra encore de la mort des trois fils de Géryon, tyran d'Espagne, où Hercule installe son fils Hispalus. Le clergé mérovingien crée le héros troyen Francus, mais sur les tapisseries de la cathédrale de Beauvais, où Francus salue la fille du Roi des Gaulois qu'il va épouser, Jupiter est représenté aux côtés d'Hercule, qui apporte à la Gaule l'alphabet de la civilisation $^{42}$.

Universidad de Valladolid

Henar Velasco López

42 J. SEZnEC, Los dioses de la antigüedad en la Edad Media y el Renacimiento, Madrid, 1983 (Paris, 1980), p. 29, 33 et 39; cf. VollKommer, o.c. (n. 20), p. 95. 